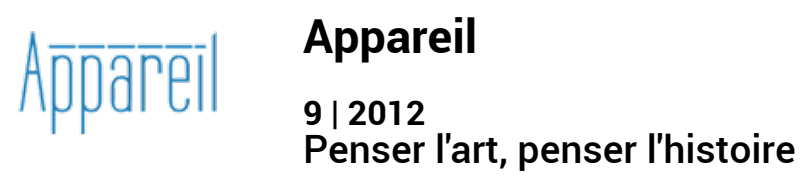

\title{
Présentation
}

\section{Audrey Rieber}

\section{(2) OpenEdition}

Journals

Édition électronique

URL : http://journals.openedition.org/appareil/1476

DOI : 10.4000/appareil.1476

ISSN : 2101-0714

Éditeur

MSH Paris Nord

\section{Référence électronique}

Audrey Rieber, «Présentation », Appareil [En ligne], 9 | 2012, mis en ligne le 02 juillet 2012, consulté le 22 septembre 2020. URL : http://journals.openedition.org/appareil/1476 ; DOI : https://doi.org/ 10.4000/appareil.1476

Ce document a été généré automatiquement le 22 septembre 2020

\section{(c) (i) (2) $\Theta$}

Appareil est mis à disposition selon les termes de la Licence Creative Commons Attribution - Pas d'Utilisation Commerciale - Pas de Modification 4.0 International. 


\title{
Présentation
}

\author{
Audrey Rieber
}

1 Penser l'art, penser l'histoire ne signifie pas seulement considérer l'art dans l'histoire, c'est-à-dire le comprendre comme un phénomène culturel qui exprimerait ou symboliserait son époque. Ce motto signifie aussi que l'inscription de l'art dans l'histoire pose des problèmes théoriques spécifiques. Aussi évidente que puisse paraître la démarche de l'historien d'art, la contextualisation historique des productions artistiques n'est pas sans engager des partis pris théoriques, discursifs, normatifs, idéologiques. En plus de rendre possible une clarification des méthodes de l'histoire de l'art, l'examen épistémologique de ces choix permet de reconsidérer certaines des catégories avec lesquelles on pense habituellement l'art et l'histoire : le style, l'œuvre, la norme, d'une part, les influences, l'évolution, le progrès, etc., de l'autre. Il n'est pas évident, d'un point de vue philosophique, d'appréhender l'objet d'art sous l'angle historique. Nombreux sont les discours à lui être consacrés qui se déploient indépendamment de cette question (pensons à l'esthétique ou à la phénoménologie). Dans quelle mesure alors penser l'art dans l'histoire, avec l'histoire, permet-il d'en dégager certains aspects spécifiques? Mais aussi, et inversement, dans quelle mesure penser l'histoire avec l'art, à partir de l'art, favorise-t-il la réélaboration de notre conception usuelle du temps?

2 Ce sont ces questions qu'affrontent les neuf contributions de philosophie et d'épistémologie de ce numéro thématique. Quatre d'entre elles sont les conférences remaniées d'un workshop consacré aux concepts fondamentaux de l'histoire de l'art ("Bildbegriffe der Kunstgeschichte", eikones NFS Bildkritik, Bâle, mai 2010). Deux articles ont été traduits de l'allemand vers le français, façon de nous inscrire dans la tradition du dialogue franco-germanique, si important lorsqu'on envisage le rapport entre histoire, art et philosophie.

3 Les trois essais liminaires ressortissent de l'épistémologie de l'histoire de l'art. Le premier est consacré au philosophe et théoricien Friedrich Theodor Vischer, tenant de cette théorie de l'empathie encore trop peu connue en France. La traduction des réflexions que consacre Hubert Locher au rôle de l'Einfühlung dans la fondation de l'histoire de l'art au XIX ${ }^{e}$ siècle était d'autant plus nécessaire. Jan Blanc, pour sa part, 
s'attaque de front aux conditions de possibilité du discours historique en interrogeant le fondement linguistique des catégories stylistiques qu'il invente et propage. "Comment dire l'art?", demande-t-il, posant dans sa radicalité la question de l'articulation entre visualité et discursivité. En entreprenant d'étudier la filiation entre les désormais incontournables Visual Studies et l'iconologie, Ralph Dekoninck met au jour l'ancrage idéologique et historiquement déterminé de notre relation à l'art et aux images. On voit donc comment, en abordant respectivement l'objectivation du subjectif, les rapports entre langage et image et entre image et idéologie, ces contributions dépassent d'emblée le champ de la méthodologie de l'histoire de l'art pour affronter quelques-uns des problèmes clés de la pensée sur l'art.

4 Partant de l'analyse d'une notion d'abord propre à l'art et à son histoire (le baroque), Anne Sauvagnargues expose la dimension à la fois descriptive (morphologique) et évaluative (tératologique) des catégories stylistiques pour, in fine, étudier le travail des normes. Muriel van Vliet s'intéresse, elle, aux procédés mis en œuvre par l'histoire de l'art, dans la mesure où ils aident à comprendre les méthodes de ces sciences historiques que sont les sciences humaines. Avec Cassirer et Foucault, elle cherche à voir en quoi l'histoire de l'art peut être un paradigme de la logique herméneutique propre aux sciences de la culture.

5 La contribution de Thomas Kisser pense, sous un autre angle encore, les correspondances entre art et histoire en examinant ensemble les logiques picturale et religieuse d'une époque donnée, mais sans réduire leur lien à une histoire des influences. Partant de la description de deux œuvres du Titien, il parvient à établir comment des concepts sont historiquement au travail dans l'image, sans méconnaître la spécificité visuelle des ouvrages de peinture. La question sous-jacente de la relation de l'art aux autres sphères de la culture est également posée par Jean-Louis Déotte qui indique la façon dont l'appareil artistique Merz lève l'opposition entre les domaines de la communication et de la publicité d'une part, et des arts, de l'autre. Parce que l'œuvre de Schwitters annonce la fin de l'ère de la surface d'inscription (la lettre, le signe, n'y ont plus pour fonction la désignation ou la dénotation), son analyse apporte aussi des éléments au thème récurrent de la discursivité.

Audrey Rieber et Clara Pacquet, enfin, retournant la conception spontanée des rapports entre art et histoire qui est de penser le premier à partir de la seconde, s'essayent à penser l'histoire à partir de l'art. La première s'attache à établir comment la prise en compte de l'art préhistorique ébranle les catégories temporelles de l'histoire. Peut-on encore envisager le déroulement historique en termes d'évolution, de téléologie et de progrès après la découverte de Lascaux ? Est-il encore possible de l'appréhender selon un axe linéaire, continu et chronologique après le choc de Chauvet? Analysant le sol classique, Clara Pacquet montre que la stratification historique du sol de Rome constitue un défi théorique tant pour les philosophes à la recherche d'un principe anhistorique d'explication de l'expérience artistique que pour les historiens d'art qui s'efforcent d'ordonner le cours de l'histoire. Dans quelle mesure le sentiment historique des artistes du XviII ${ }^{e}$ siècle fournit-il une clé à ce problème?

Le secrétariat de rédaction de ce numéro a été effectué par Lucile Cayzac 


\section{AUTEUR}

\section{AUDREY RIEBER}

Ancienne élève de l'École normale supérieure de Lyon, agrégée de philosophie, docteur en philosophie, Audrey Rieber s'intéresse principalement à l'art et à l'histoire qu'elle questionne à partir de la tradition philosophique (esthétique, philosophie et théorie de l'art, philosophie des formes symboliques, herméneutique, archéologie de Foucault, Kunstwissenschaft, Bildwissenschaft) mais aussi des concepts et méthodes de l'histoire (de l'art) : théorie des fondateurs (Vasari, Winckelmann, Burckhardt), iconologie (Panofsky), formalisme (Hildebrand, Riegl, Wölfflin), Kennerschaft, psychohistoire (Warburg), iconologie analytique (Arasse), École des Annales. Postdoctorante au sein du pôle de recherche eikones NFS Bildkritik (Bâle), elle a publié en 2012 Art, histoire et signification. Un essai d'épistémologie d'histoire de l'art autour de l'iconologie d'Erwin Panofsky (L'Harmattan, coll. Esthétiques). Elle est professeur invitée du Master « Aisthesis. Historische Kunst-und Literaturdiskurse » (KU Eichstätt, Allemagne). 